\title{
OMU: An Ontology For Mosul University
}

Ban Sharief Mustafa

Banmustafa66@uomosul.edu.iq

\section{Ibrahiem Adnan}

Brahim1995adnan@gmail.com

Department of Computer Science,

College of Computer Science and Mathematics,

University of Mosul, Mosul, Iraq

Received on: 26/03/2019

Accepted on: 27/06/2019

\begin{abstract}
Nowadays, data from different real-time data streams are coming in. The classical relational database systems cannot manage such big data. Big data should be managed in a way that keeps the semantic relations between different concepts. Ontology is a powerful tool that comes from the concept of the semantic web, can formulate data schema as semantically connected objects. Ontology can be shared and reused across different domains and sites. In this paper, an ontology that captures the main concepts and their relationships in Mosul university is proposed. The main steps for OMU (ontology for Mosul University) development are showen using protégé ontology editor. Also, several queries are implemented to show how we can use inference engine to infer new and implicit knowledge from ontologies. visualization tools are used to visualize OMU ontology.
\end{abstract}

Keywords: Ontology, Owl, University Ontology, Mosul University, Protégé

\section{OMU}

$$
\text { كلية علوم الحاسوب واليراضيات } 1 \text { جلوم إبراهيم عدنان }
$$$$
\text { بان شريف مصطفى }
$$

تاريخ قبول البحث: 2019106127

تاريخ استلام البحث: 2019\03|26

\section{الملخص}

تعتبـر شـبكة الويـب الحاليـة خاليـة مـن اي مضــمون معنـوي اذ انهـا تـربط صـفحات الويـب المخزنــة

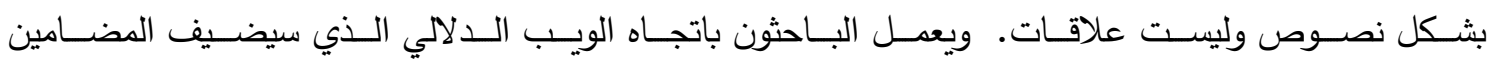

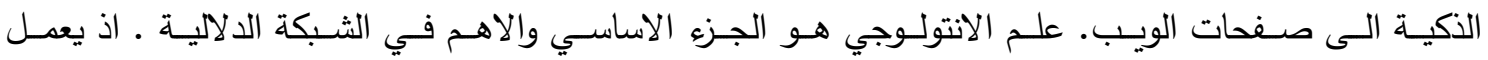

$$
\text { على توفير واجهة ذكية من المفاهيم والعلاقات بين هذه المفاهيم لتطبيقات الويب الدلالي. }
$$

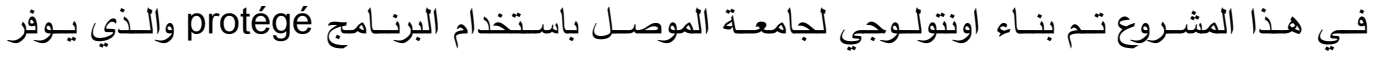

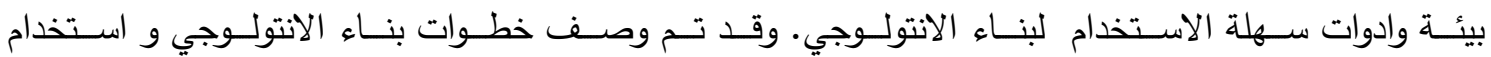

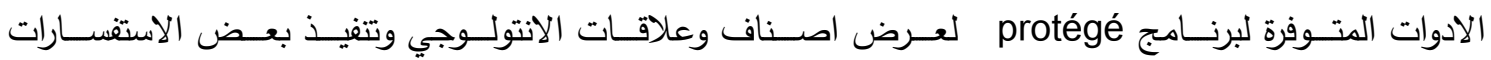




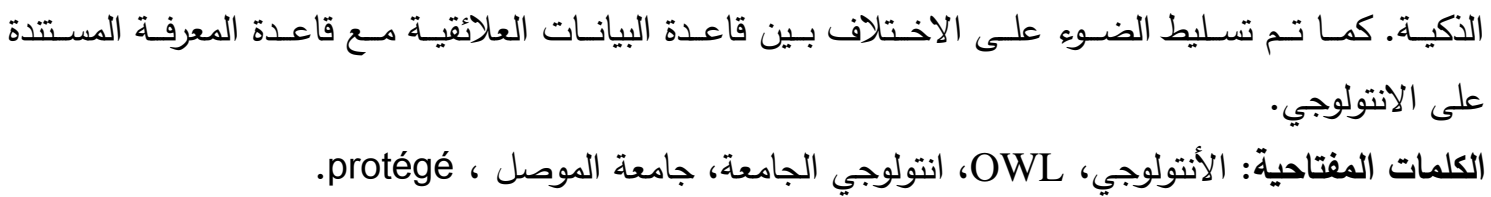

\section{Introduction}

Due to the rapid growth in data and the emergence of Big Data, there is an urgent need to adopt new approaches to manage and analyze large data. Traditional database systems are no longer able to manage big data due to its inability to deal with complex and scalable requirements. They are not able to add semantic relationships between main data concepts and fields nor can deal with logs data flow from different real time data streams [12].

The semantic web is a modern subject that establishes the emergence of the Semantic Web. T. Berners Lee, founder of the current Web, is the first to adopt the Semantic Web concept, which had a vision for the future of the Internet by adding intelligent technologies and make them a semantic network which can conclude new knowledge and answer difficult queries [2]. The semantic web project is still in its infancy, but many languages and tools have been developed to improve, expand and standardize this project. One of the most important languages developed to construct the data structure is the Web Ontology Language (OWL) language. This language is usually used in the construction of the ontology in different domains, but at present it finds more adaptation in information systems and data management [3]. It gives the possibility of structuring the data structure hierarchically and finding the semantic relations between the basic concepts, adding business rules as a higher semantic level. The use of the ontology covers the modern and expanding requirements for applications that require data management. The most important challenge comes from the importance of obtaining an integrated and non-contradictory structure for the particular domain.

In this research, an ontology in the domain of higher education and universities, based in Mosul University, has been built. The protégé program was used in the construction of the ontology. The steps to build the ontology have been made widely known, and how complex and intelligent queries for inferring a new knowledge from the ontology. The visualization tools attached to the protégé program were used to visualize OMU ontology. We try to clarify the difference between traditional database system and the ontology-based information system.

\section{Related Works}

Many researchers developed an ontology in the field of education. A research published by Malviya and Mishra [6] developed a university ontology based on Technical University (Rajiv Gandhi) in Bhopal, India. In this research, the parts and molecules of the university were explained through the University Ontology. The researcher focused on the composition of the use of protégé. The researcher focused on how to construct the ontology and some of the basic steps are documented.

Dwivedi \& Kumar [3] took the generalized structure of Indian universities and the flow of work for the development of the ontology by describing the hierarchy of classes and presenting a visual outline of the ontology. The researcher also explained the ontology's ability to perform intelligent inquiries in order to retrieve information.

Prof. Sami Abunaser\& Rasha Abdullah [1] developed an ontology for Palestine University. The research shows the difference of views and pyramid of the main and 
sub-types, the creation of a subclass, cases to clarify the layers, and the process of retrieving the query using the unified process of building UPON.

Hadjar, Kreem \& Chanane,N. [4] presents the researcher's efforts to establish university ontology. All the researcher efforts went to the development of the University ontology of Ahla University in Bahrain. In this ontology all the departments that fall under the structure of the university and the relations between them were described. The researcher developed OWL version of the old and added new types and relationships to fit the organizational chart of the University of Ahla to obtain the final OWL of the ontology. The OWL file is then imported to the Braid Top Composer to obtain a more powerful data retrieval program and to obtain the required data from the ontology easily with short SPARQ queries.

Satyamurty C.V.S., Murthy J.V.R. \& Raghava [9] developed Ontology in higher education (geometry) using the Protégé 4.3 instrument. Resource links are provided in the form of graphs OWLViz, and the or der of the class is evaluated by Fact ++. Finally, execute the semantic query to retrieve knowledge.

Last, Shahzad M. [11] in his master thesis gives the solution to the development of an ontology in the field of education and take university as example. The use of protégé to develop ontology and applying queries over it are presented.

OMU ontology have been developed based on Mosul university structure and concepts and can be extended to build an ontology-based information system for all Iraqis' higher universities [13].

\section{Ontology}

In computer science and information science, an ontology is an official representation of knowledge through a set of concepts in the field and relations between those concepts and is used to express the characteristics of this field and its description. Since the early 1990s, the ontology has become a research topic in artificial intelligence, including knowledge engineering, natural language processing and knowledge representation. Recently, the concept of Ontology has also become common in areas such as intelligent information integration, information systems, information retrieval, e-commerce and knowledge management [3].

The protégé program is used to construct the components of the ontology [6]. It is independent of the applications you use. It provides ease in the construction of the ontology and the maintenance of knowledge. Most ontology describes individuals (situations), categories (concepts), attributes, and relationships. Common components of the ontologies include [8]:

- Individuals: situations or things.

- Categories: concepts, types of objects.

- Features: aspects, properties or parameters that objects (and categories) can contain.

- Relationships: ways in which individuals and groups can communicate.

- Limitations: The formal description of what must be true until some additions are accepted as inputs.

- The axioms: assertions (including rules) in a logical form that together form the comprehensive theory that is described by the ontology in their domains.

\section{Web Ontology Language (OWL)}


OWL is a semantic Web language supported by W3C. OWL comes in three different versions: Lite, DL, and Full. OWL Full can be seen as an extension of RDF, as it accommodates all OWL and RDF language features. It maintains the maximum expression and grammatical freedom of the RDF. The problem with Full OWL is that it does not have accounting guarantees for use with the Induction program [5].

OWL DL contains all OWL language combinations, but has limitations in the use of language combinations and RDF features. For example, a class cannot be a condition for another category. It is designed to support Description Logic and has desirable properties for the inference program. OWL Lite is a set of basic OWL features, such as basic classification and key features. It aims at simplicity and ease of adoption [5]

\section{Reasoner}

Inference engines are software applications that draw new facts from existing information. Inference rules allow for the extraction of new data from already known data. Thus, new parts of knowledge can be added based on prior knowledge. By creating a model of information and relationships, we allow users to draw logical conclusions based on the model [7].

The use of semantics in the semantic web allows applications to inquire about the reason for reaching a particular conclusion, that is, semantic applications can provide evidence of their conclusions. The effects of inference illustrate the steps involved in logical thinking. In OWL-DL-based knowledge, the inference engine will be based on DL logic, and there are many common inference drivers available such as: FaCT ++, Hermit, Racer or Pellet [7].

\section{Comparison Between the Ontology and the Relational Database}

There are many similarities and differences between the ontology and the relational database. Ontology is the construction of a knowledge base based on descriptive logic and the semantic concept is crucial and important. The database is based on the relational model in the storage and retrieval of data and semantic concept is not important. Another difference is that the ontology reuses existing ones, while a new database must be created for each application domain [10]. Another point of difference lies in the way knowledge is represented: ontology uses the open world assumption, while the database operates on a closed world assumption [10].

\section{Development of OMU: Ontology of Mosul University}

The development of OMU used OWL-DL language under protégé ontology editor. The organizational structure on Mosul university has been taken for the development of ontology. The following are the main steps for the OMU construction:

Classes and class hierarchy

The main classes of the university are defined. The class hierarchy shows the subclass and superclass relationships between these classes. Fig.1 shows classes hierarchy for OMU ontology.

In ontology a class can be defined as a subclass of other class using the axiom rdfs:subClassOf. Another way to define a named class by defining a restriction using the axiom owl:equivalentClass. For example, class professor defined as subclass from class Faculty member. A restriction defines an equivalent class to professor class by the restriction:

Faculty_Member and have_an_upgrade value "assist_professor"

All individuals satisfy the above restriction belong to professor class implicitly. 
object properties between classes

Object properties define the relationships between classes individuals. These properties have characteristic which shows the type of relation that relates a class individual to another class individual.

In OMU, several object properties have been proposed to reflect meta data over our data. Fig. 2 shows some of these properties. Each property could have a characteristic that makes it work in different ways.

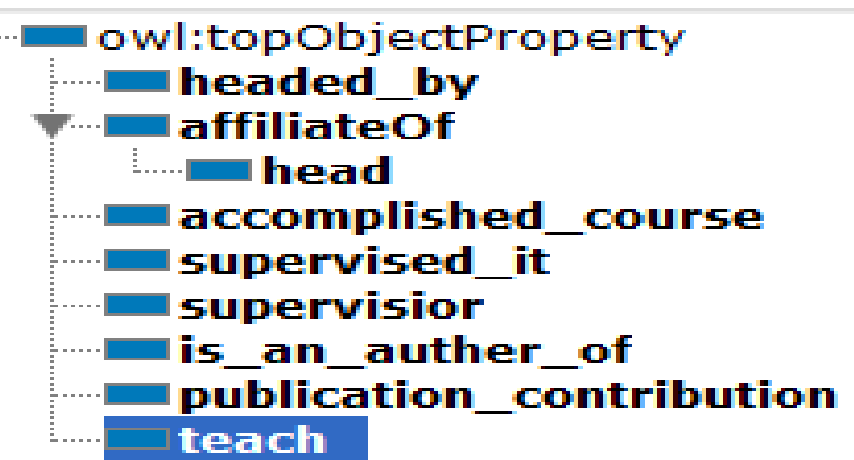

Fig. 2 Object properties in OMU ontology 


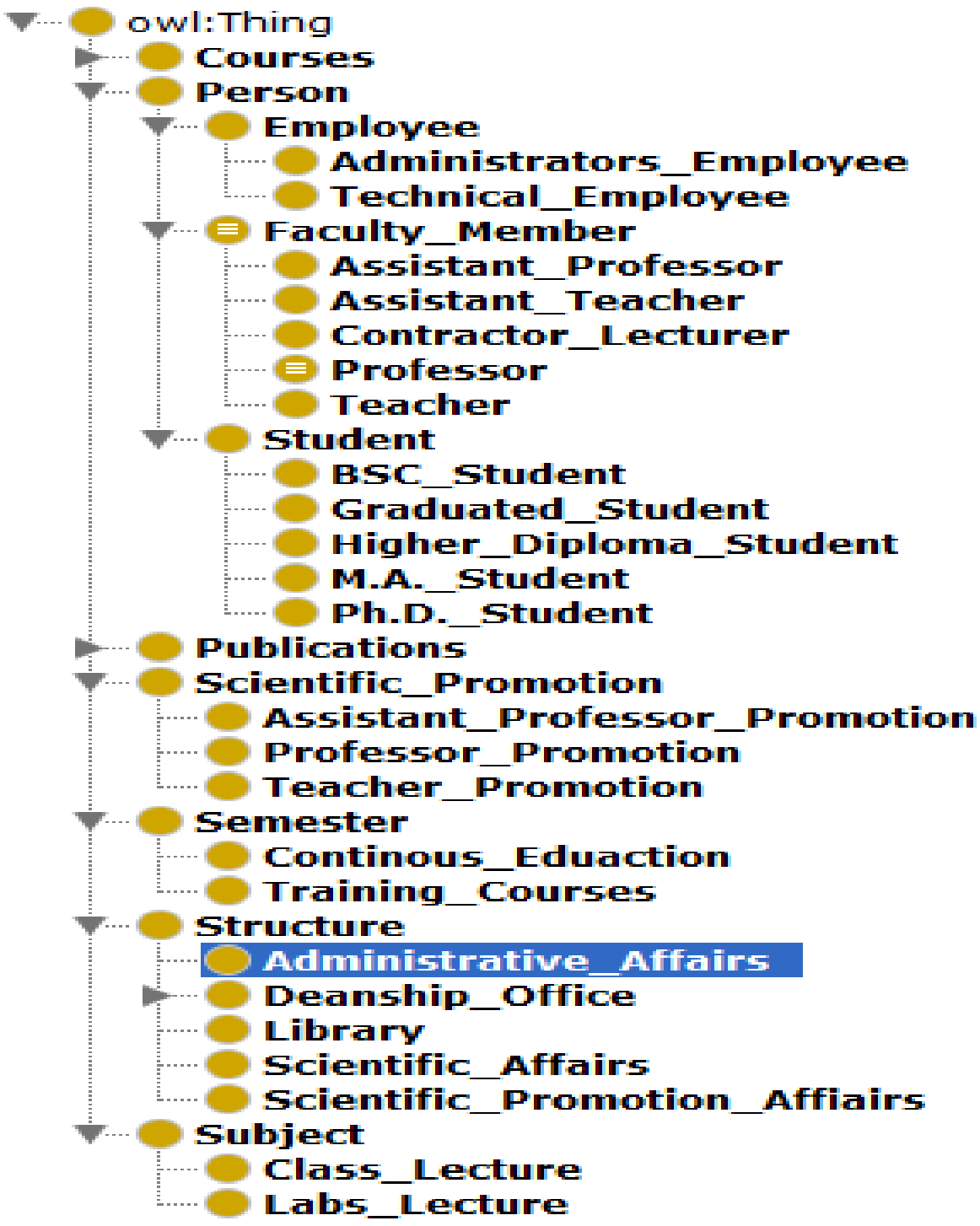

Fig. 1 OMU class hierarchy

For example, Fig. 3 shows the usage of property head:

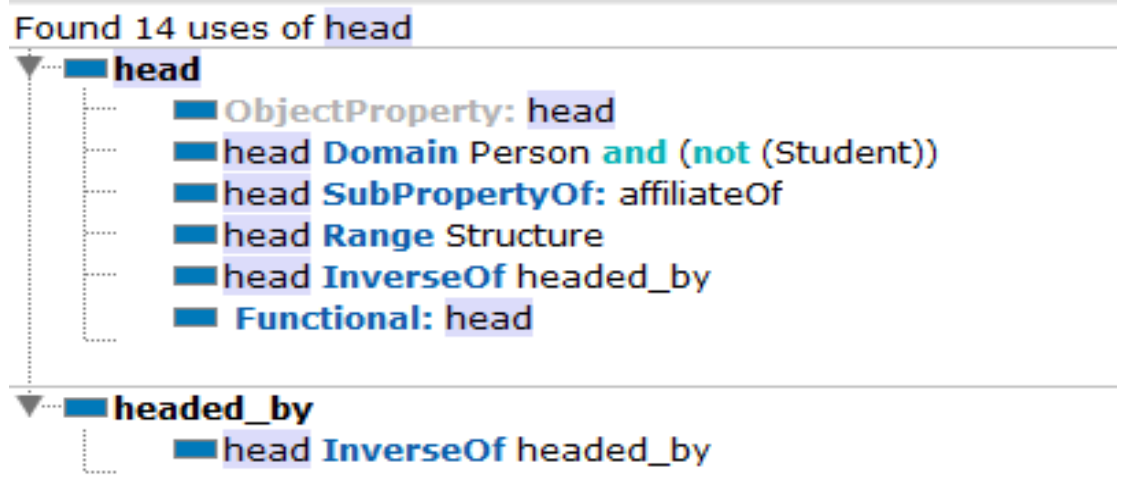

Fig.3 Head property usage

This property has functional characteristic, which means that every unit has just one administrator. The axiom sub Property Of shows that Head is sub property from 
affiliate Of property. Axiom Inverse Of defines it as an inverse relationship with headed by. The domain of the property from class Faculty Member. The range from class structure. When we define an individual to be head some unit, then ontology will assert a new fact which the inverse of the other as this unit is headed by this individual.

Data properties between classes and data items

Data property shows the relationship between classes individual and their data literal.Fig.4 shows OMU data properties. For example, sponsored_by relate a conference or workshop to some responser value string.

The Axioms of Ontology

There are two types of axioms in ontology:

- Concept axioms: These axioms usually represent the subclass and the equivalence class relationships. The axioms of class are the relationship of the class with the other classes or properties or individuals.

- Individual axioms: the axioms of individuals are the properties of the object and the characteristics of the data type related to each object.

In table 1, some of properties' axioms are listed [3]:

Table 1. Axioms With Their Syntax[3]

\begin{tabular}{|l|l|l|}
\hline S. No. & Relations & Syntax \\
\hline 1. & Relation of inclusion & rdfs:subPropertyof \\
\hline 2. & Equivalent & owl:equivalentProperty \\
\hline 3. & Inverse & owl:inverseOf \\
\hline 5. & Limitation of Function & owl:FunctionalProperty \\
\hline 6. & Inverse Function & owl:InverseFunctionalProperty \\
\hline 7. & Relation of Symmetry & owl:SymmetricProperty \\
\hline 8. & Transitive & owl:TransitiveProperty \\
\hline
\end{tabular}




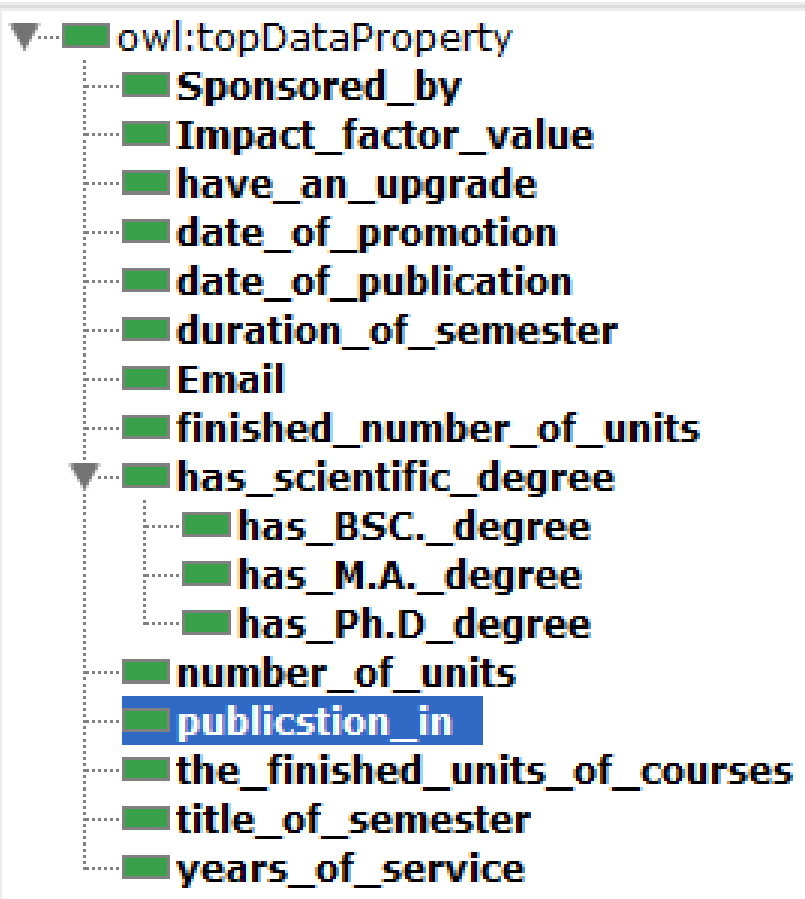

\section{Result}

\section{Fig. 5 OMU data properties}

Visualizing the Ontology

Ontology can be visualized by using protégé tools for visualization:

- Using the OntoGraph tool to display some of the main concepts and their relationships, as shown in Fig.6 which displays the class person with its other classes' relationships.

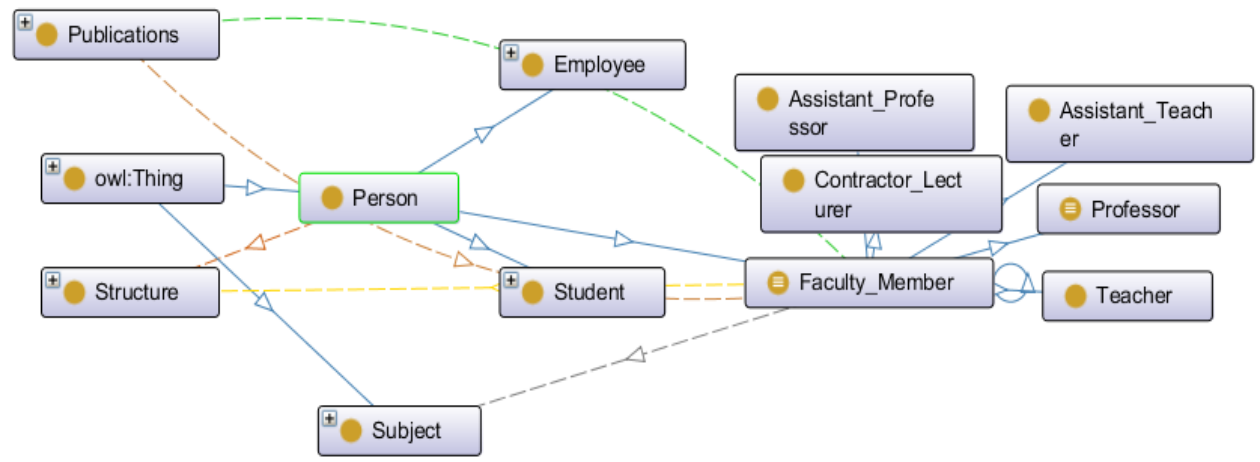

Fig.6 Person class \& its relationship by ontoGraph

- Using VOWL tool to display the complete ontology with all relations as in Fig. 7 . 


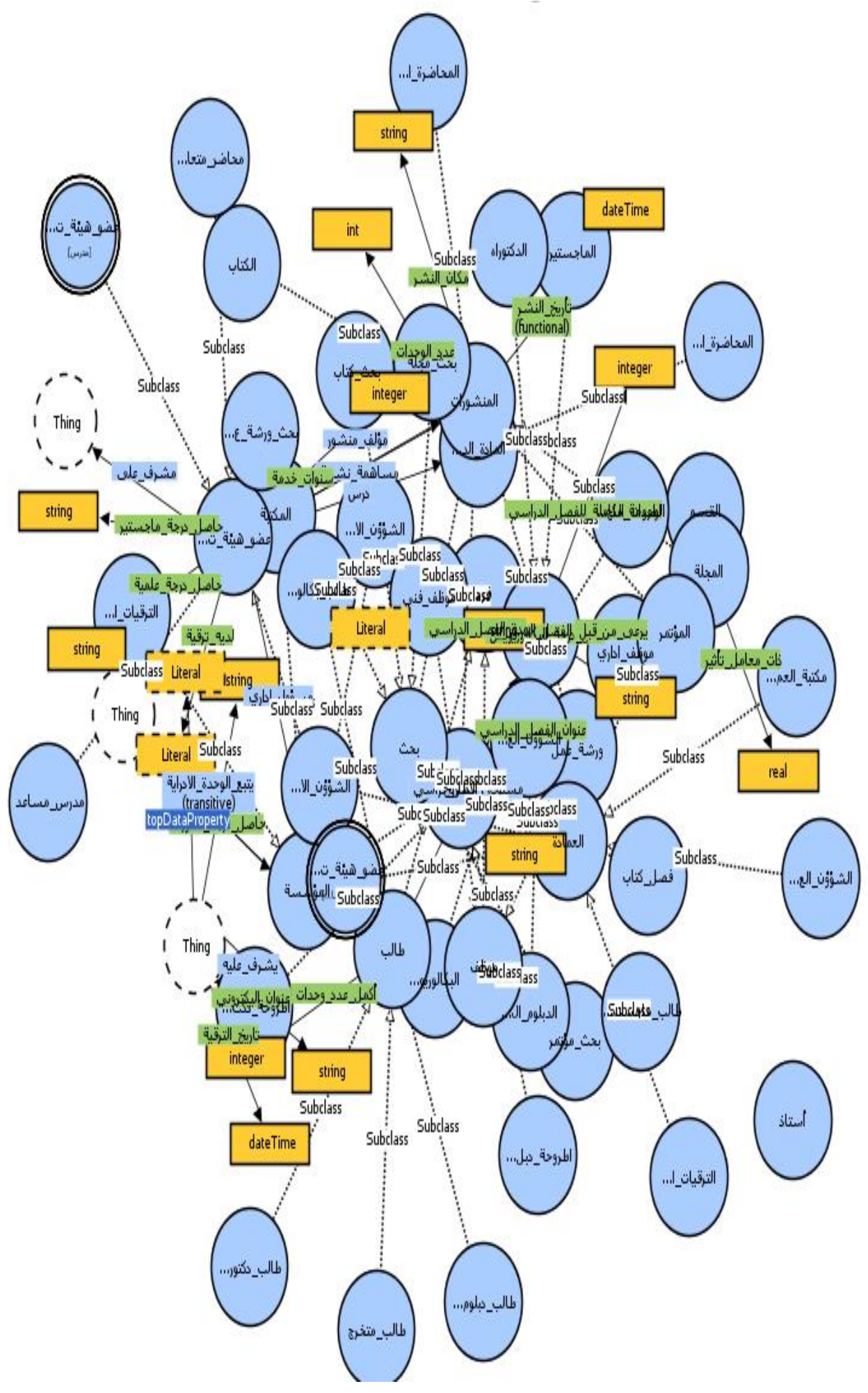

Fig. 7 OMU ontology by VOWL tool

\section{Query implementation}

The queries have a very important role in the construction of the ontology. ontology is often constructed depending on the type of query required or the amount of knowledge required. In protégé there is a tab for the query named DL Query. 
In follows some queries implementations presented to differentiate the Query in ontology from relational SQL query language:

1- Given facts/data:

Individual :Ayad_alazi.(Faculty_member)

Individual:Computer_Science(Dept.)

Facts:head (Ayad_alazi,Computer_Sciene)

Facts:affilitaeOf(computer_scine,Computer\&Mathmetics_College)

DL_Query:how affiliate_to Computer\&Mathmatics College?

As query in ontology works as inference engine, the output will add Ayad_alazi as member in this college, given two axioms: head is subproperty of affiliateOf property and affiliateOf is transitive relationship. Fig. 8 shows the query implementation.

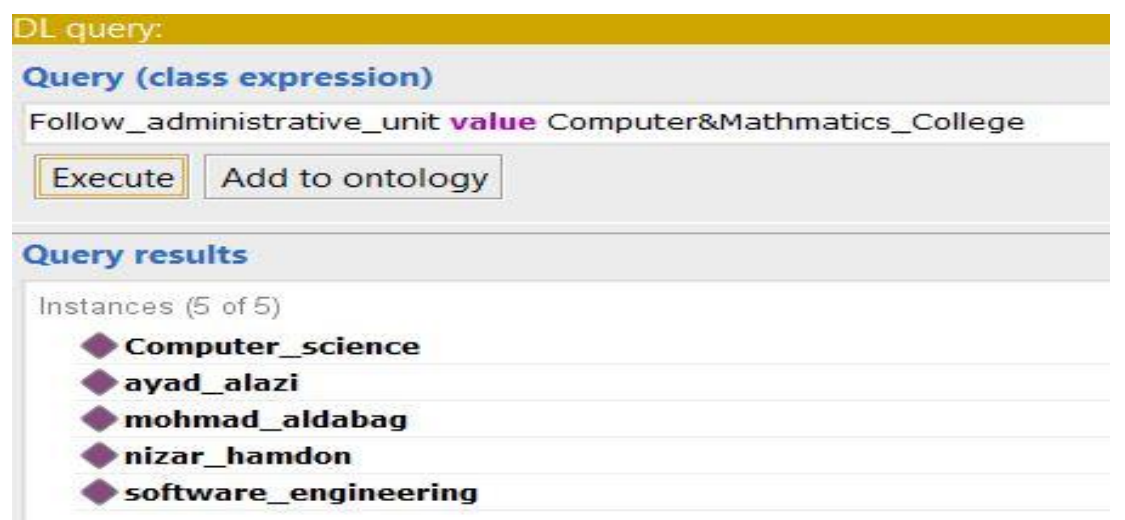

Fig. 8 Query1 implementation

1- Given facts/data:

Individual :Najla_aldabgh.(Faculty_member)

Individual:An_OntologyBased_AccessControl(Ph.D thesis)

Facts:supervisor(najla_aldabgh,An_OntologyBased_AccessControl)

DL_Query: which thesis Najla supervised?

The query use supervisor inverse characteristic with supervised _by to deduce the answer as in Fig.9

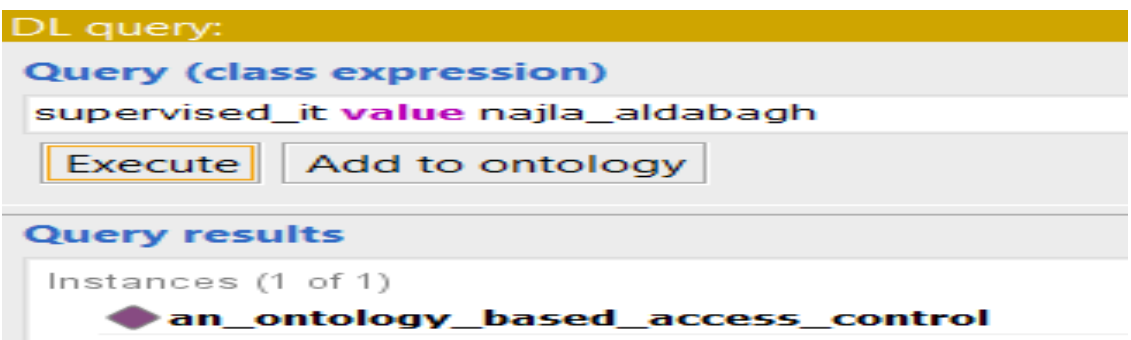

\section{Conclusion}

Fig. 9 Query 2 implementation

In this paper, we show how to construct an ontology-based information system for Mosul university. Adding semantic meaning by constructing a conceptual model for particular domain can make data management more powerful, sharable and reusable so it can be scaled over scalable platform. Queries are answered according to DL logic axioms. Thus, a new knowledge can be added always. The main challenge is building a consistent and complete ontology for domain in use. 


\section{REFERENCES}

[1] Abu Nasser, Samy and Rasha Atallah, eds. (2015)" Building an Ontology in Educational Domain Case Study for the University of Palestine "International Journal of Research in Engineering and Science Vol3.Issue1.

[2] Berners-Lee,T., Hendler, J., and Lassila, O.eds. ,(2001) "The Semantic Web", Scientific American, pp. 28-37.

[3] Dwivedi, Sanjay k. and Anand Kumar, eds. (2013)" Development of University Ontology for aSPOCMS"Journal of Emerging Technologies in Web Intelligence Vol5.No3.

[4] Hadjar K. (2016) "University Ontology: A Case Study at Ahlia University". In: Workman M. (eds) Semantic Web. Springer, Cham.

[5] Horridge,m, (2011) " Apractical guid to building owl ontology using protégé 4 and CO-OD”.

[6] Malviya, Naveen and Nishchol Mishra, eds. (2011) "Developing University Ontology using protégé OWL Tool Process and Reasoning." International Journal of Scientific \& Engineering Research Vol2.Issue9.

[7] Mustafa, Ban and Aldabagh, Najla (2014) "OJADEAC: An Ontology Based Access Control Model for JADE Platform" International Journal of Advanced Computer Science and Applications(IJACSA), 5(5).

[8] Ontology Components, http://en.wikipedia.org/wiki/Ontology components

[9] Satyamurty C.V.S., Murthy J.V.R., Raghava M. (2018) "Developing Higher Education Ontology Using Protégé Tool: Reasoning". In: SatapathyS. ,BhatejaV., Das S. (eds) Smart Computing and Informatics. Smart Innovation, Systems and Technologies, vol 77. Springer, Singapore.

[10] Sir, Michal \& Bradac, Zdenek \& Fiedler, Petr."Ontology versus Database.", (2015), IFAC (International Federation of Automatic Control) Hosting by Elsevier Ltd.

[11] Shahzad, Muhammed and Jan Nytun,eds. (2013) " A semantic web approach for dealing with university courses", Project report for Master Thesis in University of Agder, 166.

[12] Shields, Anne, (2014)" why traditional database systems fail to support big data." MARKET REALIST, https://marketrealist.com/2014/07/traditionaldatabase-systems-fail-support-big-data

$$
\text { الحاء دالة معرفية لجامعة الموصل, مشروع التخرج للمرحلة الرابعة, جامعة الموصل/قسم علوم }
$$

